\title{
Summary from an observational perspective
}

\author{
John S. Gallagher, III \\ Dept. Astronomy, U. Wisconsin, 475 N. Charter St. Madison, WI 53760, USA \\ email: jsg@astro.wisc.edu
}

\begin{abstract}
The meeting "Near Field Cosmology with Dwarf Elliptical Galaxies" covered a wide range of topics relating the general issues associated with galaxy formation and evolution as well as problems which are specific to spheroidal dwarfs. This paper summarizes these presentations in the form of an annotated guide to the presenters and their subjects. The results of the meeting demonstrate the value of focused, quantitative research on small galaxies as a way to enhance our understanding of how internal processes, cosmological foundations, and environmental conditions effect galaxy evolution.
\end{abstract}

Keywords. galaxies: dwarf, galaxies: evolution, galaxies: formation, galaxies: stellar content, galaxies: structure, galaxies:clusters: general, Local Group, dark matter

\section{Introduction}

The extreme nature of spheroidal dwarfs became immediately clear with the discovery of the Sculptor and Fornax dSph systems in the late 1930s (see Shapley 1939, Baade \& Hubble 1939). Early studies of the Virgo cluster with the Lick astrograph led G. Reaves (1953) to recognize the surprising existence of a substantial population of low luminosity Sculptor-type galaxies in the cluster and in the process provide the first appearance of the term "dwarf galaxy" in a title listed by the NASA ADS. However, it was only with the advent of fast Schmidt telescopes that the extensive populations of low luminosity dwarfs became clear. Swiss astronomer Fritz Zwicky used the 18-inch Palomar Schmidt to find large number densities of dwarf galaxies and thus demonstrated their cosmic importance (Zwicky 1957). Holding this meeting in Switzerland then was especially appropriate for many reasons, including as a reminder (especially on the centennial of Einstein's 1905 paper) that size and importance are not always correlated.

Soon after their discovery, the value of spheroidal dwarfs for stellar population studies was recognized by Baade (1944). Because of their low stellar densities and deceptively simple central structures, the $\mathrm{dE}$ and $\mathrm{dSph}$ galaxies provided insights into the characteristics of old stellar populations and formed a basis for defining the properties of Population II stars (Baade 1951). By the time the DDO catalog was published (van den Bergh 1959) dwarf galaxies were a recognized component of the nearby universe. In his influential review at the Yale symposium on stellar populations, van den Bergh (1977) further remarked on the division of dwarfs into two major groups-the old spheroidal systems and star-forming dIs. This provided an additional stimulus to understand how the populations of dwarf galaxies evolved (see also the pioneering review by Hodge 1971).

The direction of dwarf galaxy research took a new twist with the advent of models in which galaxies contain dark matter. Precision stellar radial velocity dispersion measurements by Aaronson (1983) showed that some dSphs have higher apparent mass-to-light ratios than can be accounted for by normal stellar populations, thereby opening the way for observational support of cold dark matter (CDM) models. The companion paper by Lin \& Faber (1983) initiated explorations of the wider astrophysical implications of dwarf galaxies whose mass is domnated by CDM. 
In this meeting we have heard discussions that center on spheroidal dwarfs but extend over topics ranging from star formation to cosmology. There is no easy way to coherently summarize the diversity of subjects. In $\S$ II I present the main topics of discussions followed by lists of contributors, so that this summary might serve as an annotated guide to the program and the proceedings. A few concluding remarks are included in $\S$ III.

\section{Major Discussion Points}

\subsection{Dwarfs in the Youthful Universe}

Dwarfs \& Cosmology - Colafrancesco, Zhao: The survival of dSph galaxies with their low stellar densities as neighbors of giant galaxies is a long standing problem. One possibility is that the existence of such objects is a signature of non-Newtonian gravity, such as the MOND models of gravitational interactions. It therefore was useful to hear a discussion of the issues that arise and paths towards their solution in placing MOND in a cosmological context by including relativity. On the other side, the current standard model for low density dwarfs is that they are bound within CDM halos. In this case these objects have high central phase space densities of CDM. This and the proximity of the Galactic dSphs make them interesting targets for efforts to directly detect particle emission associated with possible annihilation processes in CDM.

Formation of Dwarf Galaxies - Diemand, Mashchenko, Ricotti: Numerical models of mass particles which interact only via gravity now have sufficient resolution to describe the evolution of moderate mass bound structures within a cosmological context. These models, when run for standard density perturbation spectra in $\Lambda$ CDM cosmologies, predict large numbers of low mass 'mini-halos' clustered around the large halos which presumably contain giant galaxies. How one interprets this excess is a key question: If dSphs occupy what originally were relatively low mass DM halos, then the number of luminous dwarfs per giant galaxy is far less than the expected number of halos. This in turn leads to a fundamental issue that is illustrated in Figure 1: How do we connect the observable stellar galaxies to the models of DM halos; are we even talking about the same objects?

Impact of Reionization - Grebel, Suza: DSph galaxies contain ancient stars and yet evolved to the point where they produce mean metal abundances above $1 \%$ of solar. Since such galaxies, or related classes of low mass objects that merged rapidly into giant galaxies, should have been very common, they are potentially important contributors to the reionization of the universe. On the other hand, in some models reionization profoundly influences the evolution of low mass galaxies to the point where it can cut off or cut down the available amount of ISM and thus truncate star formation. Unfortunately, modern stellar population studies have yet to detect any signature of a preferred epoch for the cessation of star formation in nearby dSphs. Instead the star formation histories appear to vary with galaxy location, suggesting that local factors determine the evolutionary paths of these systems.

Dwarfs in the Distant Universe? - Barazza, Ferguson, Pettini: In the past decade we gained a remarkable level of access to the youthful universe. Galaxies now have been reliably observed up to $z \approx 6$, or at a point where only $\sim 1$ Gyr had elapsed since the big bang. The compact sizes of galaxies observed with $H S T$ at $z \geqslant 2$ suggest that some connections with present day dwarfs is possible if some dwarfs are born via extreme starbursts, where a significant fraction of their stars are produced in a galactic crossing time of $\sim 100$ Myr. However, the star formation histories of most nearby dwarfs suggest they did not evolve in this way, but rather formed stars more gradually over a time scale 


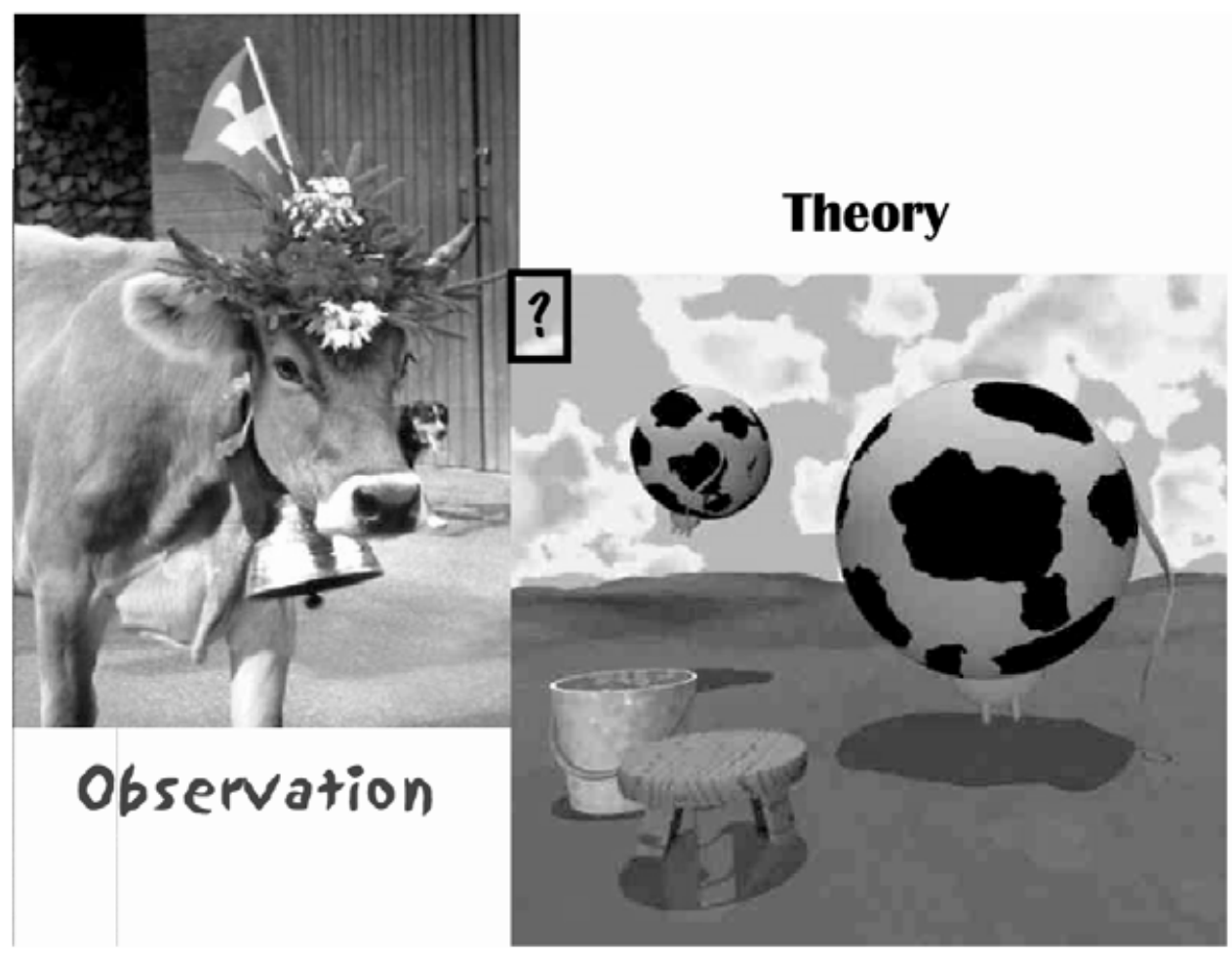

Figure 1. A key point is how to compare theoretical models and observations. While the same words are used, observers and theorists are not necessarily describing objects in the same ways, as illustrated by the above descriptions of Swiss cows by observers and theorists.

of several Gyr. Unfortunately, the present studies of galaxies at high redshifts have yet to turn up any objects that are unambiguously dwarfs. Perhaps studies of low luminosity Ly $\alpha$ emission line sources might be one way to find the high redshift ancestors of present day dwarf galaxies?

\subsection{The Stellar Populations}

Modeling the Birth and Evolution of Stellar Populations - Dekel, Hensler, Lin, Marcolini, Michielsen, Weidner: We face a fundamental difficulty in that galaxies, with the exception of cases where an object is found via gravitational lensing alone, are the only known tracers of moderate mass scale cosmic structures that are bound by gravity. Galaxies contain baryons and these in turn make stars. Thus the complex processes of gas capture into galaxies and the subsequent feedback from stellar populations, as well as external affects, must be taken into account when producing models of galaxies vs. models of dark halos. The ability of gas to change phases and interact with the host galaxy in many ways, including gravity, collisional processes, photoionization, and via magnetic fields complicates the critical problem of modeling the baryons. In dealing with dSphs we face the further difficulty that some mechanisms must exist to cause star formation to cease and the remaining system to be almost free of any cool gas. Further progress in "gastrophysics," the study of the flow of mass and energy through the ISM and IGM associated with galaxies, remains essential. However, even in the absence of a 
full physical understanding of the many physical links between gas and stars, models are developing in predictive power and providing important guides to observations, such as the increased emphasis in understanding the role of mass outflows and inflows of both gentle and violent types.

Reading the Stellar Populations - Arimoto, Da Costa, Gallart, Gullieuszik, Harbeck, Held, Hempel, Koch, Miller, Rejkuba, Saviane, Tolstoy: The advent of the Hubble Space Telescope in combination with large ground-based telescopes has allowed us to extend "Galactic astronomy," the study of galaxies through the properties and dynamics of their stellar populations, throughout the Local Group and beyond. One key set of observations show that the lower stellar mass function is relatively normal in Galactic $\mathrm{dSphs}$; there is little room for extra stellar mass in dSphs in the form of very low mass stars.

The interpretation of Hess diagrams, stellar densities on color-magnitude planes, has become a developed art that yields information on stellar abundances and star formation histories. These studies reveal the complex nature of star formation in even the dSphs dominated by old stellar populations. As is appropriate for galaxies, a range in metallicity exists, radial stellar population gradients are seen, and extended periods of active star formation are derived. This work also highlights differences between the Galactic and M31 dSph satellites; the Milky Way has the younger, more diverse companions while the M31 dSphs are predominantly older stellar fossils. It also demonstrates the existence of a smooth evolutionary continuum between the various classes of dSphs, and "transition" dwarf galaxies, such as Phoenix or LGS3, which appear to share stellar population features with the younger dSphs, like Fornax. On the other hand, the dSphs and transition dwarfs appear to have had higher initial star formation rates than the dIs, indicating that some of the differences among dwarfs go back to early times.

Work on the chemical evolution of dwarfs also is experiencing a boom powered largely by high and medium resolution spectroscopic studies carried out at the VLT and Keck Observatories. Consistent with models based on Hess diagrams, the Galactic dSphs show depressed $[\alpha / \mathrm{Fe}]$ relative to Galactic halo stars, implying that enrichment took place over a longer time. Evidently the Galactic halo is not primarily composed of stars that were stripped from mature dSph galaxies. On the other hand, the detection of very extended old stellar populations around some irregular galaxies supports the idea that basic structural similarities exist among all classes of dwarfs.

The Gas Crises - Dekel, Marcolini, Skillman: This topic was widely discussed across the meeting, but was not the main focus of an individual contribution. The main point is that dSphs are the most gas-deficient class of galaxy; they have no detectable ISM (see Young 2000, Gallagher et al. 2003). How the ISM in these galaxies was lost is not yet established, but a combination of internal (shell break-outs, photoionization) and external (tides, ram pressure stripping) factors are likely to be involved. The issue of gas retention connects to one distinction between star-forming dwarfs, which tend to be widely distributed within and beyond galaxy groups, versus the dSphs/dEs that are clustered within groups. No field dSph galaxies currently are known, suggesting that the conditions leading to gas removal are intimately tied to galaxy groups and clusters.

\subsection{Environmental Connections}

Satellite Galaxies - Chilingarian, Coleman, Evans, Kroupa, Lee, Mateo, Read, Prugniel, Wilkinson, Zaggia: A key problem in dSph astronomy is how these objects manage to survive, and what their original nature might have been. Are dSphs tracers of the full population of low mass dark halos, tidally trimmed remnants of galaxies in moderate mass halos, or systems that are not in simple equilibrium and therefore have no 
dark matter halos? The answers to these questions go beyond dwarf galaxy research and constrain the nature and amount of dark matter. The difficulty in understanding the structures of dwarfs on the observational side arise from the problems in studying their outer regions, and from our inability to measure internal proper motions, thus leaving us with only projected radial velocities. Here we saw that the outermost radial velocity dispersion profiles are still being debated, as are the forms of the density profiles. The structural issues are illustrated by the rather smooth Draco system, and clumpy, somewhat distorted Ursa Minor dSph, and yet both galaxies have similar kinematics. Thus the amount and radial distribution of dark matter-cusp or core structure-remains uncertain.

Dwarf-Giant Connections - Bailin, Font, Gallagher, Ibata, Kroupa, MartinezDelgado, Sohn: There is no longer any doubt that dwarfs feed the halos of giants. The Sagittarius stream is one such long-lived remnant of tracing stellar contributions to the Milky Way's halo. The issue is how common could such streams be, and how many should we expect to observe if the bulk of the halo was produced from dissolved dwarfs? Realistic models show that early tidal debris is randomized as a giant galaxy forms around it, while the later streams can survive for several wraps. We also need to keep in mind the baryonic mass contributions in the form of gas that is being made to the Milky Way by the Magellanic Clouds, and was likely also captured from dSphs in their youth. However, for a dwarf companion that is in a prograde orbit near the disk plane of a spiral, there also is the possibility of substantial gas capture from a tidally induced spiral arm. Thus the gas flows between dwarfs and their hosts may go two ways, as can gravitational interactions, e.g., by dwarfs exciting warps in their spiral hosts.

Another feature in the dwarf giant connection is the Holmberg effect, where companion galaxies are in either the disk or polar directions. Furthermore, within the the polar cone, where most of the Milky Way's dSphs lie, the systems seem to be nearly coplanar. Its not clear how this feature of the Galactic dSphs fits with CDM dwarf models.

Locating Dwarfs - Dunn, Jerjen, Karachentsev, Rizzi, Rekola: While the low stellar densities of NGC 147 and NGC 185 allowed Baade to resolve their red giants in the mid-1940s, these properties now permit accurate distance measurements to dwarfs out to and beyond $10 \mathrm{Mpc}$ from the luminosity of the tip of the red giant branch. This is done directly via resolved stars with HST and indirectly via surface brightness from the ground. The results presented here show that we are entering an era where dwarf galaxies can be prized distance markers, a process that soon should be extended as adaptive optics (AO) techniques improve on large ground-based telescopes.

Membership in Galaxy Groups - Da Costa, Grebel, Karachentsev, Mateo, McConnachie, Skillman, Taylor: The populations of dwarf galaxies in the Local Group are statistically sharply divided with the early-type systems mainly (but not entirely!) clumped around the Milky Way and M31, while the dI galaxies spread throughout the Local Group. Within the Local Group we can look for finer details; how do the properties of distant and nearby dSphs compare in terms of star formation histories, gas content, structures, etc.? Is there a "typical dSph galaxy"? The general spatial distribution of dwarfs seems to be repeated elsewhere and so such detailed studies are likely to apply beyond the Local Group. Yet the M31 dSphs, as well as those in other galaxy groups have different mean properties than the Milky Way satellites. Whether these properties are imprinted through initial conditions or the later environment is difficult to sort out as dwarf galaxies are mostly cosmically immobile; they stay near where they were formed.

Evolutionary Transitions - Debattista, Drinkwater, Evistigneeva, Graham, Hwang, Kayser, Knezek, Mayer, Prugniel, Scannapieco, Read, Vaduvescu: At one level we are asking whether the evolutionary paths of dwarfs are primarily set by properties at formation or by later events-a classic nature vs. nurture issue. On another we explore how, 
when, and why low mass galaxies should cease being active star formers and drop into the inactive dwarf category. A third issue is the connection, if any, between rotationally supported and non-rotating classes of dwarfs. Models show that a variety of opportunities exist in nature to modify the structures of galaxies: tides, gas loss or accretion, dynamical heating through collisions, mergers, and the effects of external ionizing radiation fields, to name a few. We therefore expect that some fraction of present day dwarfs will have been shaped by their environments.

What about the observations? There is no doubt that the population of dwarfs continues to experience secular evolution. This can be seen in features such as tidal and gas streams from satellite dwarfs to their wide range in stellar population ages. We also find galaxies whose properties place them between the disky Irrs and triaxial dEs, such as the apparently non-rotating NGC 5253 starburst dwarf. Ultracompact dwarfs (and possibly closely related peculiar globular stars clusters, such as $\omega$ Cen) may be the ultimate examples of galaxies whose natures have been changed via interactions; it is very unlikely that the structures of these types of galaxies bear much resemblance to their initial states.

Galaxy Clusters - Caldwell, Conselice, Coté, DeRijcke, Hilker, Lisker, Kovac, Mastropietro, Matkovic, Popesso, Rakos, Tully, van Zee, Zibetti: Galaxy clusters are the densest galactic environments and also the places with the largest populations of dwarfs. The two broad explanations for this phenomenon are that small halos containing stars were better able to survive in clusters and thus give us a true picture of dwarf galaxy numbers (which are closer to the expectations of CDM models), or that dwarfs in clusters of galaxies are remnants of a variety of types of galaxies that were trimmed down through interactions within the cluster. This is another area where the past decade has seen a substantial shift in position as the inhomgeneous nature of cluster $\mathrm{dE} / \mathrm{dSphs}$ populations was recognized. Some spheroidal dwarfs are rotationally flattened, and others are evidently intrinsically triaxial; a range of stellar population ages exist; and at a given luminosity there also are spreads in metallicity and surface brightness.

It seems unlikely that either the dwarf by birth or dwarf by transformation will explain everything and some combination of processes is likely. For example, differing rates of the intrinsic evolutionary clocks can lead to stellar metallicity and age differences due solely to internal factors. If the majority of dwarfs are perturbed descendants of other classes of galaxies, then large numbers of these objects must have fallen into galaxy clusters. These were primordial star forming dwarfs in pictures where the transformations are from lateto-early type dwarfs, or moderate luminosity disk systems if the transformations were more severe. In either case the process of whittling down galaxies to make dwarfs would contribute intracluster baryonic matter, gas and stars. Properties of the intracluster stars (ages, metallicities, spatial distributions) will be very useful in constraining dwarfby-transformation models for galaxy clusters.

The comparison of luminosity functions as a function of galaxy density suggests the existence of a trend for steeper luminosity functions in denser locations. However, even the steepest luminosity functions for spheroidal dwarfs are not as steep as the predictions for illuminated low mass halos from standard CDM models.

\section{Concluding Thoughts}

Bruno Binggeli introduced the meeting by briefly touching on several key issues: the degree of unity of $\mathrm{dSph} / \mathrm{dE}$ galaxies as a structural class, the degree to which the fair samples from the local universe complement far field cosmological studies, the apparently prominent position of dark matter in dwarf galaxies, and the role that such systems play 
in the overall process of galaxy formation. That we can even ask and quantify these questions is a measure of the degree of progress in this field.

While most of the questions rate a "work in progress" status, we can answer the point about the homogeneity of the $\mathrm{dSph} / \mathrm{dE}$ classes. Neither is homogeneous as both classes show a range of kinematic and stellar population properties. Perhaps we can make progress by viewing these types of galaxies as being structural endpoints that are reached by a variety of evolutionary paths accessible to low density, gas poor galaxies?

We also are still in a difficult triangle bounded by the expectations for large numbers of dwarf satellite halos (and therefore dwarf galaxies?) from CDM models, the uncertain dynamical states and structures of satellite dwarf galaxies, and the difficulties in dealing with the evolution of baryons from capture into galaxies and eventual formation of stars. We can look for continued improvements in several key areas, e.g., as more accurate radial velocities and proper motions become available (and a potential leap forward via GAIA and SIM). Furthermore, in addressing these issues through studies of dwarf galaxies we have the advantages of numerous objects spanning a range properties that are nearby. We therefore expect work on these visually unimpressive objects will continue to contribute in major ways to our understanding of galaxies and the state of matter in the universe.

\section{Acknowledgements}

I would like to thank the organizers for producing an excellent meeting and for providing a full week of Alpine photometric weather. My research on dwarf galaxies is supported by NASA through grants administered by the Space Telescope Science Institute in conjunction with U.S. general observer programs, and by the National Science Foundation through grant AST-9803018 to the University of Wisconsin.

\section{References}

Aaronson, M. 1983, ApJ 266, L11

Baade, W. 1944, ApJ 100, 147

Baade, W. 1951, Publ. U. Michigan Obs., vol. 10, p. 33

Baade, W. \& Hubble, E. 1991, PASP 51, 40

Bergh, S. van den 1959, Publ. David Dunlop Obs., vol. 2, p. 147 (No. 5)

Bergh, S. van den 1977, in: B. M. Tinsley \& R. B. Larson (eds.), The Evolution of Galaxies and Stellar Populations (New Haven: Yale Univ. Obs.), p. 19

Gallagher, J.S., Madsen, G.J., Reynolds, R.J., Grebel, E.K. \& Smecker-Hane, T. A. 2003, ApJ 588,326

Hodge, P. 1971, ARA\& A 9, 35

Lin, D.N.C. \& Faber, S.M. 1983, ApJ 266, L21

Reaves, G. 1953, PhD thesis, University of California, Berkeley

Shapley, H. 1939, Proc. Nat. Acad. Sci. 25, 565

Young, L. 2000, AJ 119, 188

Zwicky, F. 1957, Morphological Astronomy (Berlin: Springer) 